\title{
Alteration in the cerebrospinal fluid lipidome in Parkinson's disease: A pilot study
}

\author{
Joaquín Fernández-Irigoyen, Paz Cartas-Cejudo and Enrique Santamaría *
}

\begin{abstract}
Clinical Neuroproteomics Unit, Navarrabiomed, Complejo Hospitalario de Navarra (CHN), Universidad Pública de Navarra (UPNA), IdiSNA. Navarra Institute for Health Research, Irunlarrea, 331008 Pamplona, Spain; jfernani@navarra.es (J.F-I); pazcarce@hotmail.com (P.C.-C.)

* Correspondence: esantamma@navarra.es; Tel.: +34-848-425-740; Fax: +34-848-422-200
\end{abstract}

\begin{abstract}
Lipid metabolism is clearly associated to Parkinson's disease (PD). Although lipid homeostasis has been widely studied in multiple animal and cellular models as well as in blood derived from PD individuals, the cerebrospinal fluid (CSF) lipidomic profile in PD remains largely unexplored. In this study, we have characterized the CSF lipidomic imbalance between neurologically intact controls $(n=10)$ and PD subjects $(n=20)$. The combination of dual extraction with ultra-performance liquid chromatography-electrospray ionization quadrupole-time-of-flight mass spectrometry (UPLC-ESI-qToF-MS/MS) allowed to monitor 257 lipid species across all samples. Complementary multivariate and univariate data analysis pointed out that glycerolipids (mono-, di-, and triacylglycerides), saturated and mono/polyunsaturated fatty acids, primary fatty amides, glycerophospholipids (phosphatidylcholines, phosphatidylethanolamines), sphingolipids (ceramides, sphingomyelins), $\mathrm{N}$-acylethanolamines and sterol lipids (cholesteryl esters, steroids) were significantly increased in the CSF of PD compared to control group. These results, despite the limitation of being obtained in a small population, demonstrate and extensive CSF lipid remodelling in PD, shedding new light on the deployment of CSF lipidomics as a promising tool to identify potential lipid markers as well as discriminatory lipid species between PD and other atypical parkinsonisms.
\end{abstract}

Keywords: lipids; cerebrospinal fluid; parkinson's disease; mass-spectrometry; lipidomics

\section{Introduction}

Due to the lipid heterogeneity, it has been estimated that the human lipidome may be composed by 100,000 different lipid species ${ }^{1,2}$. Lipids play multiple roles in brain function, affecting the elasticity and structural organization of synaptic membranes, and modulating protein activity involved in cellular signalling dynamics ${ }^{3-5}$. In the context of $\mathrm{PD}$, a genetic risk has been characterized between lipid/lipoproteins traits and the disease ${ }^{6}$. Mutations in lipid-producing enzymes (associated with familial PD; GBA) and SNPs in multiple genes involved in lipid metabolism (SREBF1, DGKQ, ASAH1, SMPD1) have been linked to sporadic PD ${ }^{7-10}$. Moreover, lipids not only influence in the aggregation potential of alpha-synuclein in vitro and in vivo ${ }^{11}$ but also are present in high concentration as components of crowded membranes, vesicle structures and dismorphic organelles present in Lewy bodies ${ }^{12}$. All these data evidence that lipid metabolism should be tightly regulated to counteract the appearance and progression of PD.

Lipidomics is emerging as a powerful approach that complements protein and gene-centric workflows in the biomarker search to evaluate the neurodegenerative risk or the neurodegenerative progression ${ }^{13}$. Although the scientific community is in a continuous learning curve to obtain a comprehensive portrait of the human brain lipidome ${ }^{14}$, the deployment of different variants of chromatographic separations coupled to mass-spectrometry is considered the gold standard approach to study lipid profiles in a high-throughput manner. However, multiple efforts are needed to solve and standard- 
ized the associated analytical challenges ${ }^{15}$. Several lipidomic platforms have been recently used to characterize the lipid composition of biofluids in neurological disorders such as amyotrophic lateral sclerosis, multiple sclerosis and Alzheimer's disease (AD) 16-20. In this study, we have applied a discovery workflow to determine the global lipidomic changes at the CSF level between PD and controls using ultra-performance liquid chromatography-electrospray ionization time-of-flight mass spectrometry (UPLC-ESI-ToF-MS), monitoring more than 250 lipid species and detecting a new metabolic signature associated to the disease that should be further validated in extensive sample cohorts in terms of biomarker sensitivity and specificity.

\section{Materials and Methods}

\subsection{Materials}

Internal standard (IS) compounds: nonadecanoid acid, dehydrocholic acid and tryptophan-(indole-d5) were purchased from Sigma-Aldrich. 1-tridecanoyl-2-hydroxy-sn-glycero-3-phosphocholine (13:0 Lyso PC), N-hexanoyl-D-erythro-sphingosylphosphorylcholine $\quad$ SM $\quad(\mathrm{d} 18: 1 / 6: 0)$, 1,2-diheptadecanoyl-sn-glycero-3-phosphoethanolamine $\quad(17: 0 \quad \mathrm{PE})$, 1,2-dinonadecanoyl-sn-glycero-3-phosphocholine $\quad(19: 0 \quad P C) \quad$ and N-heptadecanoyl-D-erythro-sphingosine Ceramide (d18:1/17:0) were purchased from Avanti Polar Lipids. Tritridecanoylglycerol (13:0 TG), Triheptadecanoylglycerol (17:0 TG) and Cholesteryl Laurate ChoE (12:0) were purchased from Larodan Fine Chemicals. All chemicals and solvents (acetonitrile, methanol, water, isopropanol, formic acid, ammonium formate) were of analytical, HPLC or HPLC-MS grade. See Appendix A for IS working solution preparations (Table A1 and A2).

\subsection{Metabolite extraction from CSF}

Control ( $\mathrm{n}=10$; mean age: 77.7 years; $4 \mathrm{~F} / 6 \mathrm{M})$ and $\mathrm{PD}(\mathrm{n}=20$; mean age: 79.9 years; 7F/13M) CSF samples were obtained from the Parkinson's UK Brain Bank funded by Parkinson's UK, a charity registered in England and Wales (258197) and in Scotland (SC037554) (Table 1). $150 \mu \mathrm{L}$ of CSF were vortexed with $600 \mu \mathrm{L}$ of ice-cold IS working solution for platform $1,30 \mu \mathrm{L}$ of ice-cold IS working solution for platform 2 and $570 \mu \mathrm{L}$ of ice-cold $\mathrm{CHCl}_{3}$. After shaking (20 min. room temperature) and incubation $\left(1 \mathrm{~h} \mathrm{at} 4{ }^{\circ} \mathrm{C}\right.$ ), a centrifugation step was performed $\left(18,000 \times \mathrm{g}\right.$ for $15 \mathrm{~min}$ at $\left.4{ }^{\circ} \mathrm{C}\right)$. For lipidomic platform $1,650 \mu \mathrm{L}$ were dried at $40{ }^{\circ} \mathrm{C}$ in a vacuum concentrator. After centrifugation $(18,000 \times \mathrm{g}$ for $15 \mathrm{~min}$ at $4{ }^{\circ} \mathrm{C}$ ), dried samples were dissolved in $50 \mu \mathrm{L}$ methanol and vortexed at room temperature for $20 \mathrm{~min}$. A centrifugation step $\left(18,000 \times \mathrm{g}\right.$ for $5 \mathrm{~min}$ at $\left.4{ }^{\circ} \mathrm{C}\right)$ was performed to precipitate any particulates, transferring the supernatant to a plate for analysis of lipidomic platform 1 . For lipidomic platform $2,650 \mu \mathrm{L}$ were mixed with $50 \mu \mathrm{L}$ of $\mathrm{H}_{2} \mathrm{O}$ and vortexed for a few seconds. After centrifugation $\left(18,000 \times \mathrm{g}\right.$ for $5 \mathrm{~min}$ at $\left.4^{\circ} \mathrm{C}\right)$, $400 \mu \mathrm{L}$ of the lower organic phase were dried at $40{ }^{\circ} \mathrm{C}$ in a vacuum concentrator. Dried samples were dissolved in $50 \mu \mathrm{L}$ of acetonitrile:isopropanol 1:1, and shaken vigorously at room temperature for $10 \mathrm{~min}$. A centrifugation step $\left(18,000 \times \mathrm{g}\right.$ for $5 \mathrm{~min}$ at $\left.4{ }^{\circ} \mathrm{C}\right)$ was performed to precipitate any particulates, transferring the supernatant to a plate for analysis of lipidomic platform 2 . 
Table 1. Chromatographic and mass-spectrometric workflows used in this study.

\begin{tabular}{|c|c|c|}
\hline & Platform 1 & Platform 2 \\
\hline Column type & $\begin{array}{c}\text { UPLC BEH C18, } 1.0 \times 100 \\
\mathrm{~mm}, 1.7 \mu \mathrm{m}\end{array}$ & $\begin{array}{l}\text { UPLC BEH C18, } 2.1 \times 100 \\
\mathrm{~mm}, 1.7 \mu \mathrm{m}\end{array}$ \\
\hline Flow rate & $0.140 \mathrm{~mL} / \mathrm{min}$ & $0.400 \mathrm{~mL} / \mathrm{min}$ \\
\hline Solvent A & $\begin{array}{c}0.05 \% \text { Formic Acid in wa- } \\
\text { ter }\end{array}$ & $\begin{array}{l}\text { Water:Acetronitrile }(2: 3)+ \\
\text { 10mM Ammonium Formate }\end{array}$ \\
\hline Solvent B & $\begin{array}{c}0.05 \% \text { Formic Acid in ace- } \\
\text { tonitrile }\end{array}$ & $\begin{array}{c}\text { Acetonitrile:Isopropanol } \\
(1: 9)+10 \mathrm{mM} \text { Ammonium } \\
\text { Formate }\end{array}$ \\
\hline$(\% \mathrm{~B})$, time & $0 \%, 0 \mathrm{~min}$ & $40 \%, 0 \mathrm{~min}$ \\
\hline$(\% \mathrm{~B})$, time & $50 \%, 2 \mathrm{~min}$ & $100 \%, 10 \mathrm{~min}$ \\
\hline (\%B), time & $100 \%, 13 \mathrm{~min}$ & $40 \%, 15 \mathrm{~min}$ \\
\hline$(\% \mathrm{~B})$, time & $0 \%, 18 \mathrm{~min}$ & $40 \%, 17 \mathrm{~min}$ \\
\hline Column temperature & $40^{\circ} \mathrm{C}$ & $60^{\circ} \mathrm{C}$ \\
\hline Injection volume & $2 \mu \mathrm{L}$ & $3 \mu \mathrm{L}$ \\
\hline Autosampler temperature & $10^{\circ} \mathrm{C}$ & $10^{\circ} \mathrm{C}$ \\
\hline Source temperature & $120^{\circ} \mathrm{C}$ & $120^{\circ} \mathrm{C}$ \\
\hline Nebulisation $\mathrm{N}_{2}$ flow & 600 L/hour & 1000 L/hour \\
\hline Nebulisation $\mathrm{N}_{2}$ temperature & $350^{\circ} \mathrm{C}$ & $500{ }^{\circ} \mathrm{C}$ \\
\hline Cone $\mathrm{N}_{2}$ flow & 30 L/hour & 30 L/hour \\
\hline Ionization & ESI -ve & ESI +ve \\
\hline Capillary voltage & $2.8 \mathrm{kV}$ & $3.2 \mathrm{kV}$ \\
\hline Cone voltage & $50 \mathrm{~V}$ & $30 \mathrm{~V}$ \\
\hline Type of data & Centroid & Centroid \\
\hline Scan time & $0.2 \mathrm{~s}$ & $0.2 \mathrm{~s}$ \\
\hline Acquisition range & 50-1000 Da & 50-1200 Da \\
\hline
\end{tabular}

Analysis of fatty acyls, bile acids, steroids and lysoglycerophospholipids was carried out with lipidomic platform 1 and analysis of glycerolipids, cholesterol esters, sphingolipids and glycerophospholipids was performed with lipidomic platform 2.

\subsection{Chromatography and mass-spectrometry}

Lipidomic profiling was carried out by OWL Metabolomics S.L. (Derio, Spain). Briefly, chromatographic separation and mass spectrometric detection conditions employed for each UHPLC-ToF-MS-based platform is indicated in Table 1. An Acquity-LCT Premier XE system and an Acquity-Xevo G2QTOF (Waters Corp., Milford, MA) were used as Platform 1 and 2 respectively.

\subsection{Data processing and normalization}

TargetLynx application manager for MassLynx 4.1 software (Waters Corp. Milford, USA) was used for data processing. A set of parameters associated to metabolites included in the analysis (Rt $\mathrm{m} / \mathrm{z}$, mass-to-charge ratio pairs, retention time)were incoporated into the program. Using a mass tolerance window of $0.05 \mathrm{Da}$ and after peak detection and noise reduction (at LC and MS levels), only true metabolite related features were processed by the software. For each sample injection, a list of chromatographic peak areas was generated. Data normalization was performed following the procedure described by Barr et al. ${ }^{21}$ where the ion intensity corresponding to each peak present in each CSF sample was normalized respect to the sum of peak intensities in each CSF sample. There were no significant differences ( $t$-test $=0.1031$ ) between the total intensities used for normalization of the sample groups compared in the study.

\subsection{Data analysis}


Once normalized, the dimensionality of the complex data set was reduced to enable easy visualization of any metabolic clustering of the different groups of samples. This was achieved by multivariate data analysis, including the non-supervised principal components analysis (PCA) and/or supervised orthogonal partial least-squares to latent structures (OPLS) approaches. Univariate statistical analyses were also performed calculating group percentage changes and unpaired Student's t-test p-value (or Welch's t-test where unequal variances were found) for the comparison between both experimental groups.

\section{Results}

During a neurodegenerative process, different type of molecules could be released and finally diffused into the CSF circuit, being considered potential cerebrospinal fluid (CSF) biomarkers. Due to cell membrane breakdown is a characteristic feature of a neurodegenerative process in brain syndromes, the deep characterization of CSF metabolomic profiles could reveal specific lipid molecules released by damaged neuronal or glial cell populations, establishing novel molecular panels to help us in the characterization of neurodegenerative diseases. In the current study, we have focused our attention on the metabolic profile of CSF lipids in PD.

\subsection{Categorization of the detected CSF lipidome}

Due to the wide concentration range of lipids and to their extensive chemical diversity ${ }^{1}$, it is not possible to analyze the full lipidomic profile in a single experiment. Therefore, lipid extraction was carried out by fractionating the CSF samples into groups of species with similar physicochemical properties, using appropriate solutions of organic solvents (methanol, chloroform/methanol) and then analyzing the different extracts in specific analytical platforms ${ }^{21}$. In our case, two UHPLC-MS based platforms were used (Figure 1) to maximize the analysis of CSF lipidomic profiles derived from neurologically intact controls and PD subjects (Table 2), performing an optimal profiling of: i) Fatty acyls, bile acids, steroids and lysoglycerophospholipids, and ii) Glycerolipids, glycerophospholipids, sterol lipids and sphingolipids. Using this dual workflow, a total of 257 metabolic features were detected in all human CSF samples, including 6 bile acids, 10 fatty amides, 3 acylcarnitines, 65 glycerolipids, 111 glycerophospholipids, 22 non-esterified fatty acids, 33 sphingolipids and 7 sterols (Supplementary Table 1). 
Table 2. CSF samples included in the lipidomic study. PD: Parkinson's disease; C: controls. Duration (years).

\begin{tabular}{|c|c|c|c|c|}
\hline SAMPLE.ID & Age & Sex & Onset & Duration \\
\hline PD354 & 88 & F & 77 & 11 \\
\hline PD423 & 66 & $\mathrm{~F}$ & 53 & 13 \\
\hline PD436 & 90 & M & 82 & 8 \\
\hline PD520 & 80 & M & 56 & 24 \\
\hline PD530 & 85 & M & 77 & 8 \\
\hline PD357 & 71 & M & 37 & 34 \\
\hline PD450 & 66 & M & 47 & 19 \\
\hline PD495 & 88 & F & & \\
\hline PD501 & 89 & F & 82 & 7 \\
\hline PD537 & 84 & M & 84 & 9 \\
\hline PD550 & 83 & $\mathrm{~F}$ & 77 & 7 \\
\hline PD562 & 79 & M & 72 & 7 \\
\hline PD636 & 84 & M & 65 & 20 \\
\hline PD295 & 83 & M & 67 & 16 \\
\hline PD340 & 67 & M & 53 & 14 \\
\hline PD356 & 86 & F & 75 & 9 \\
\hline PD541 & 72 & M & 66 & 6 \\
\hline PD546 & 84 & F & 71 & 13 \\
\hline PD579 & 76 & M & 55 & 21 \\
\hline PD591 & 77 & M & 68 & 9 \\
\hline $\mathrm{C} 022$ & 65 & M & & \\
\hline $\mathrm{C} 023$ & 78 & $\mathrm{~F}$ & & \\
\hline C030 & 77 & M & & \\
\hline C008 & 93 & $\mathrm{~F}$ & & \\
\hline C015 & 82 & M & & \\
\hline $\mathrm{C} 026$ & 78 & $\mathrm{~F}$ & & \\
\hline C032 & 88 & $\mathrm{M}$ & & \\
\hline C054 & 66 & M & & \\
\hline C064 & 63 & F & & \\
\hline C076 & 87 & M & & \\
\hline
\end{tabular}

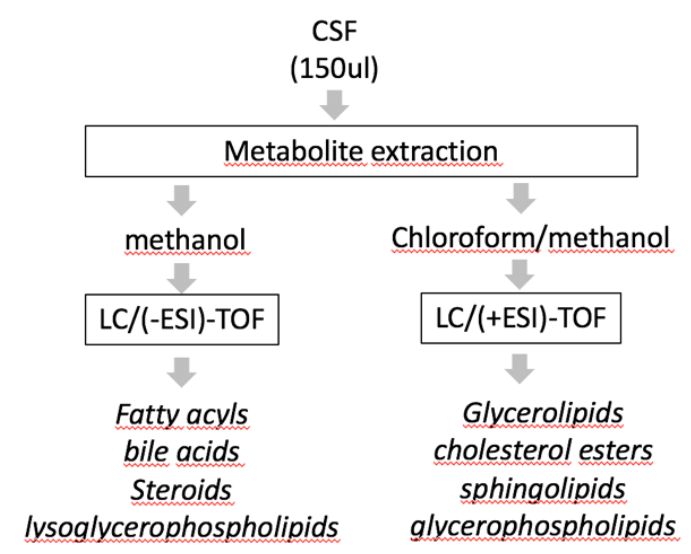

Figure 1. Lipidomic workflow applied in our pilot study.

\subsection{CSF lipidomic profiling in Parkinson's disease}

The 257 detected lipid features were analyzed across all CSF samples. Once normalized, the dimensionality of the complex dataset was reduced to enable easy visualisation of any metabolic clustering of the PD and control CSFs. The quality of the global experiment was assessed (see Appendix A). 


\subsubsection{Multivariate analysis}

A supervised OPLS model was also calculated in order to achieve the maximum separation between both experimental groups. Figure 2 (left panel) shows the score scatter plot of this model, in which a clear clustering of CSF samples according to the presence or absence of PD was observed. Similar to what was found for the Loadings scatter plot displayed in Figure A4 (Appendix A), metabolites responsible for the differences observed were mainly glycerolipids (MAG, DAG, TAG), fatty acids (SFA, MUFA), FAA, glycerophospholipids (PC, PE) and sphingolipids (Cer, SM), which were increased in PD group (Figure 2, right panel). However, this model had a low predictive ability $(\mathrm{Q} 2 \mathrm{X}=0.150)$ being necessary to extend this pilot study including additional sample cohorts.
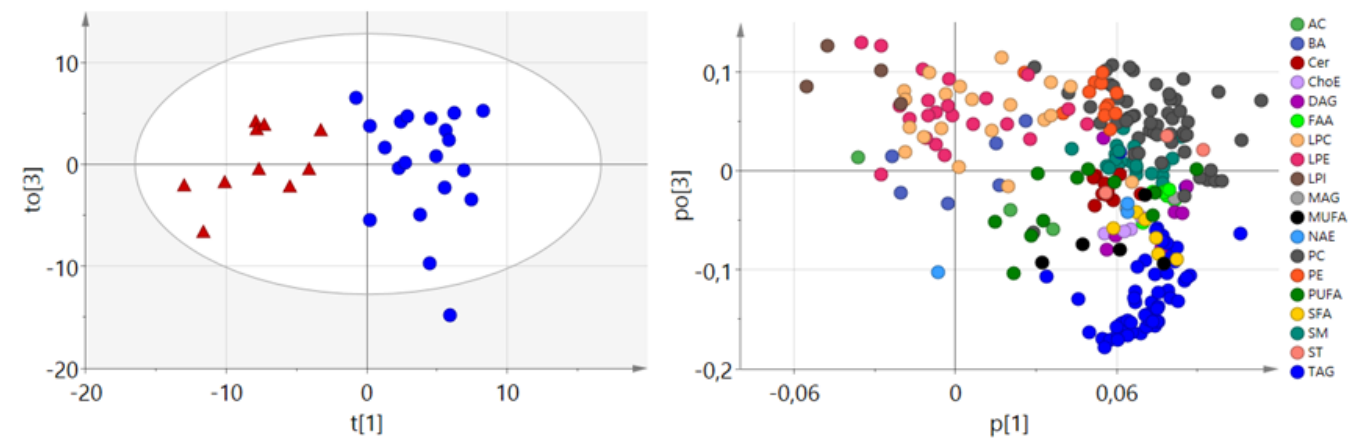

Figure 2. Score scatter plot (left panel) and Loadings scatter plot (right panel) of the OPLS-DA model of CSF samples after square root transformation of the data. Model diagnostics $(A=9 ; R 2 X=$ $0.860 \mathrm{Q} 2 \mathrm{X}=0.150$ ). red triangules and blue circles represent the ontrol and PD group respectively.

\subsubsection{Univariate analysis}

Univariate data analysis was also performed, calculating group percentage changes and unpaired Student's t-test $p$-value (or Welch's $t$ test where unequal variances were found) for the PD vs. control comparison. As mentioned in Appendix A, Shapiro-Wilk test revealed that the majority of the CSF metabolites measured from PD were not following a normal distribution. Then, in addition to the untransformed data analysis, a square root (sqrt) transformation of the data was also applied. Raw intensity data, average group intensities, fold changes and unpaired Student's t-test of each individual metabolite and of each metabolic class for both untransformed and sqrt transformed data are included in Supplementary Table 1. The raw data per metabolic class was calculated as the sum of the normalized areas of all the metabolites with the same chemical characteristics. In order to help in the visualization of the results, a heatmap was generated. The heatmap in Figure 3 displays the log2 (fold-change) of the 257 metabolites included in the analysis together with the unpaired Student's t-test for the PD vs. control comparison obtained using untransformed data (left column) and the square root (Sqrt) transformation of the data (right column). 


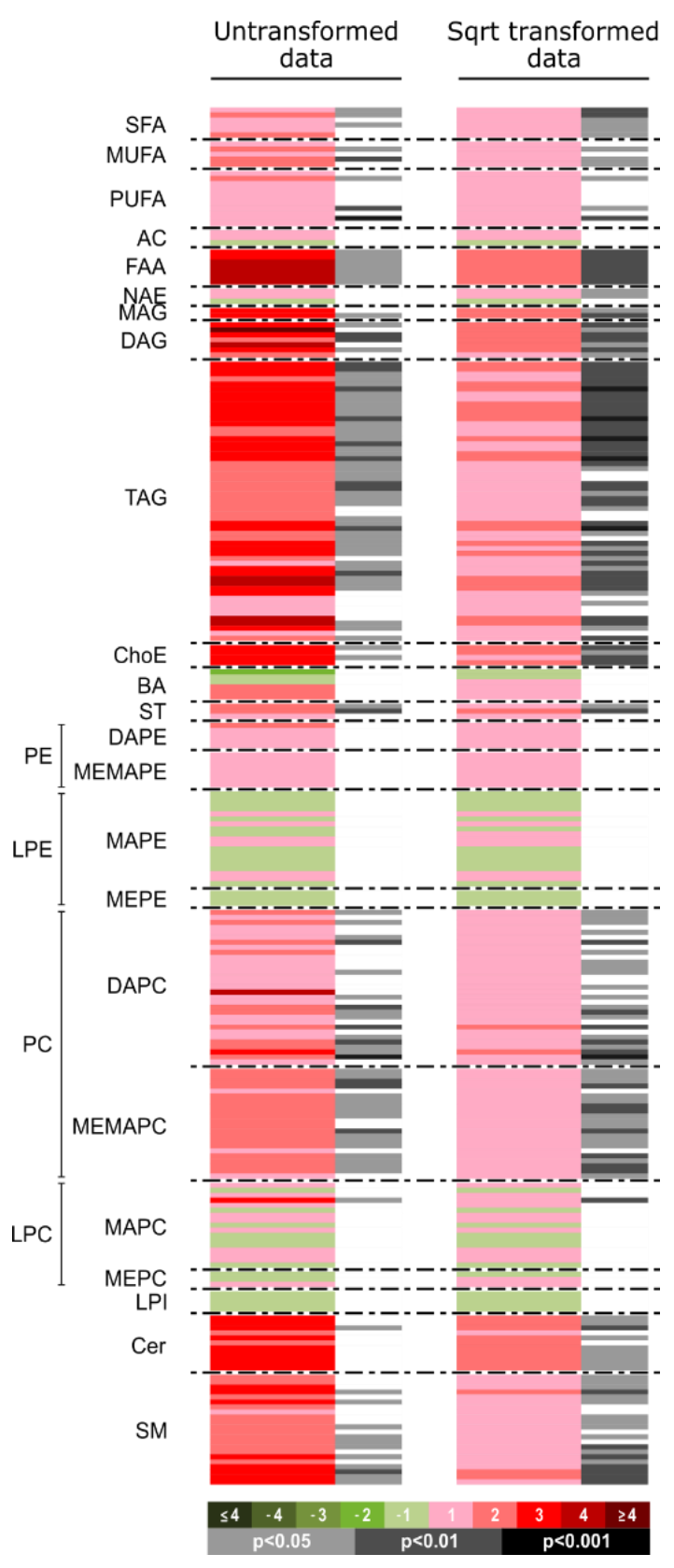

Figure 3. Heatmap representing differential individual metabolic features obtained from the PD and control comparison. Log transformed ion abundance ratios are depicted, as represented by the scale. Darker green and red colours indicate the change intensity of the metabolite levels, respectively. Grey lines correspond to significant fold-changes of individual metabolites, darker grey colours have been used to highlight higher significances ( $p<0.05, p<0.01$ or $p<0.001$ ). It is relevant to highlight that metabolites have been ordered in the heatmap according to their carbon number and unsaturation degree of their acyl chains. Heatmap colour codes for log2 (fold change) and unpaired Student's t-test p-values are indicated at the bottom of the heatmap. Metabolite order is supplied in the "Heatmap datasheet" in Supplementary Table 1.

As shown in Figure 3, a similar trend in the levels of the lipid metabolites was found when using the untransformed data or the square root transformation of the data (right column, Figure 3). Additionally, in order to complement the heatmap, a volcano plot was generated highlighting the most significant metabolites considered individually for the PD vs. control comparison (Figure 4). 


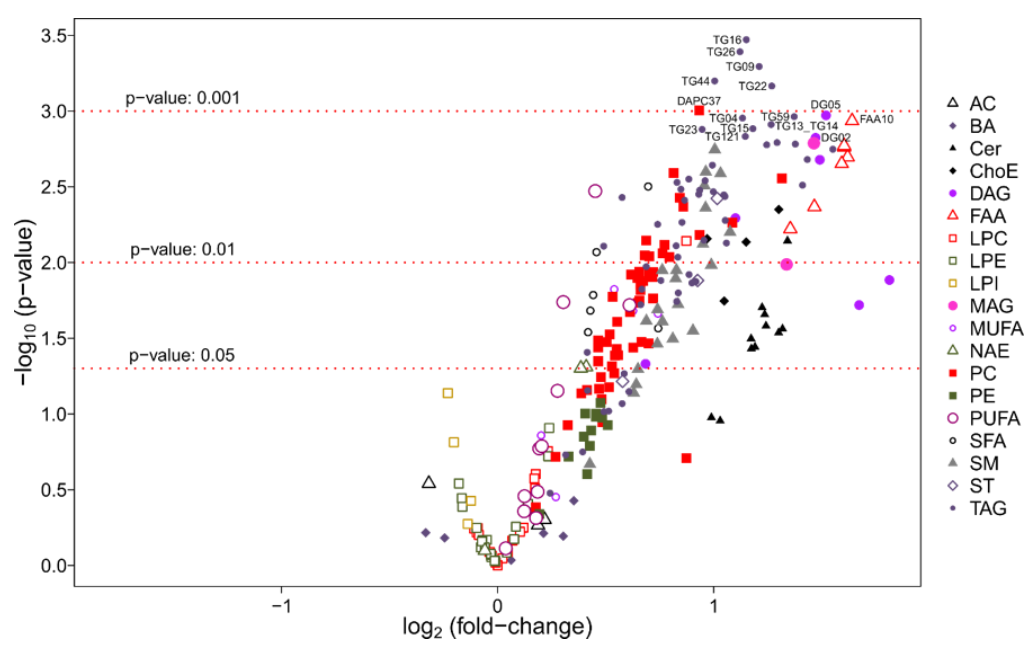

Figure 4. Volcano plot [-log10(p-value) vs.log2(fold-change)] for the PD vs. control subjects comparison. This volcano plot highlights the significance $p$-value $<0.01$ for DG02 -DG(32:0)-, DG05 -DG(34:0)-, FAA10 -22:0 amide-, TG04 -TG(44:0)-, TG13_TG14 -TG(47:0)-, TG15 -TG(47:1)-, TG23 -TG(49:1)-, TG59-TG(56:1)- and TG121 -TG(40:0)- and the significance $\mathrm{p}<0.001$ for DAPC37 -PC(22:4/20:4)-, TG09 -TG(46:0)-, TG16 -TG(48:0)-, TG22 -TG(49:0)-, TG26 -TG(50:0)- and TG44 -TG(53:1)-. The vast majority of them were glycerolipids and more specifically, triacylglycerols (TAG).

Lipid classes were also calculated as the sum of the normalized areas of all the lipid metabolites with the same chemical characteristics (Supplementary Table 1). Interestingly, all lipid classes significantly altered in PD subjects were increased. Changes in some of the most relevant metabolite classes are depicted in the boxplots shown in Figures 5 .
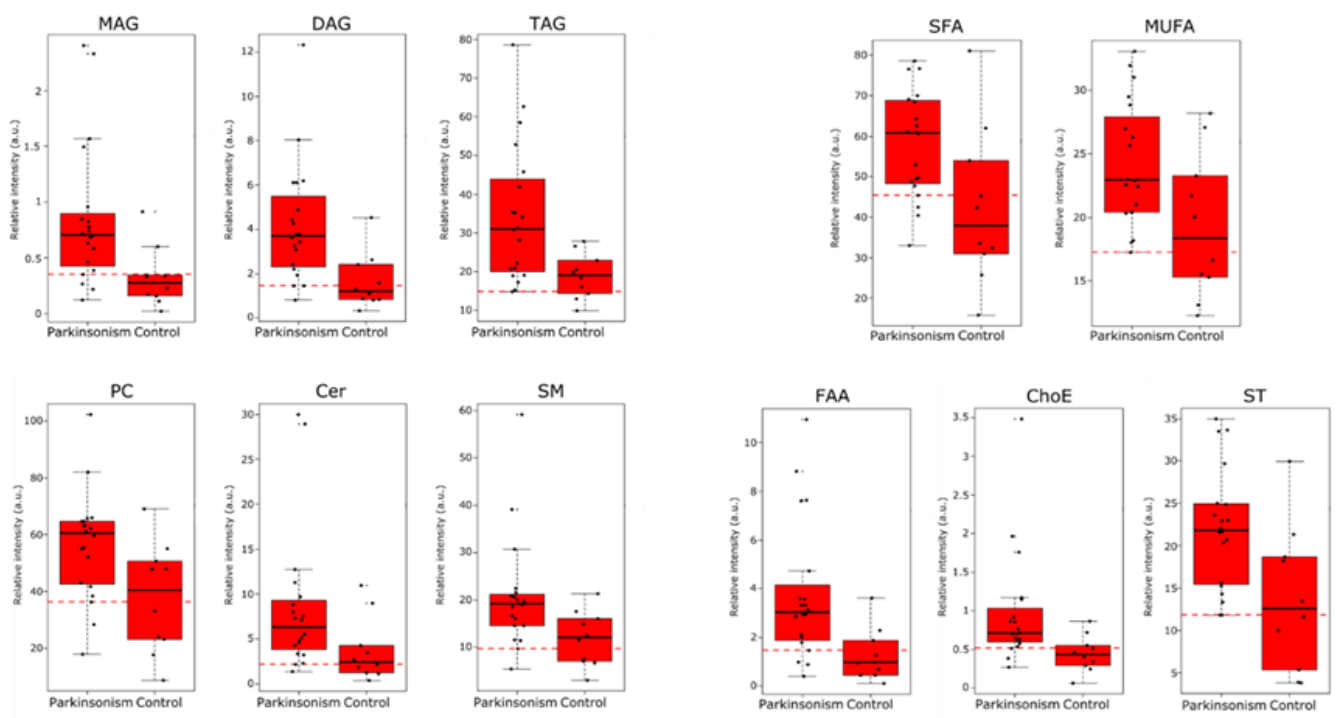

Figure 5. Boxplots of glycerolipids (monoacylglycerols -MAG-, diacylglycerols -DAG-, triacylglycerols -TAG-), phosphatidylcholines -PC- and sphingolipids (ceramides -Cer-, sphingomyelins -SM-) (left). Boxplots of non-esterified fatty acids -NEFA- (saturated fatty acids -SFA-, monounsaturated fatty acids -MUFA-), primary fatty amides -FAA- and sterol lipids (cholesteryl esters -ChoE-, steroids -ST-) (right).

\section{Discussion}

Brain lipids act as the major source of energy, provide insulation to cells and structural integrity to membranes and can be rapidly converted to signalling molecules or to inflammatory intermediates ${ }^{22}$. Thus, changes in lipid metabolism and its reflection on 
CSF lipid content might have a significant impact on brain function contributing to PD pathogenesis. Although the exact role of lipids in PD is not totally understood, the effects and/or levels of a subset of the lipidome has been partially characterized in plasma as well as in animal/cellular PD models ${ }^{23}$. However, brain levels of lipids may not correlate with plasma levels, so additional CSF measurements are needed to address the gap in knowledge about the potential pathological or compensatory composition of the brain lipidome in PD. In our case, all the lipid species which were found to be significantly increased in parkinsonian CSF were: i) Several non-esterified fatty acids (NEFA), including the complete profile of saturated fatty acids (SFA), some monounsaturated fatty acids (MUFA) and a few polyunsaturated fatty acids (PUFA); ii) various primary fatty amides (FAA) and N-acyl ethanolamines (NAE), iii) almost the complete profile of glycerolipids, including monoacylglycerols (MAG), diacylglycerols (DAG) and triacylglycerols (TAG), iv) several cholesteryl esters (ChoE) and steroids (ST), v) almost the complete profile of phosphatidylcholines (PC) and vi) the majority of ceramides (Cer) and sphingomyelins (SM).

It has been speculated that SFA could exacerbate PD pathology ${ }^{24}$. Moreover, higher SFA levels are present in frontal cortical lipid rafts from PD subjects respect to controls ${ }^{25}$. Although different CSF MUFA levels have been detected between several PD phenotypes, MUFA levels remain unchanged in the temporal cortex from PD subjects 26, 27. PUFA levels in the anterior cingulate cortex are increased in PD, although their CSF levels depend on the disease etiology 27,28 . At molecular level, PUFA and alpha-synuclein are involved in synaptic vesicle cycle ${ }^{29}$. Moreover, it has been evidenced that PUFA increase alpha synuclein oligomerization through the interaction with the N-terminal region 30,31 . Respect to glycerolipids, the exact function of MAG is unknown. While DAG is a secondary lipid messenger that plays a role in the synaptic vesicle cycle ${ }^{32,33}$, TAG is directly involved in energy storage ${ }^{34}$. In the context of PD, plasma DAG and TAG tend to be diminished in PD, and higher serum TAG have been linked to reduce risk of PD ${ }^{35-37}$. Alpha-synuclein overexpression has been directly related with intracellular TAG deposition 38,39 .

In spite of CSF alterations in several cholesteryl esters (ChoE) and steroids (ST), little is known about the impact of sterols in PD pathogenesis ${ }^{23}$. In general, sterols are known to play a role in immunity, membrane fluidity and serve as signaling mediators ${ }^{40,41}$. In $\mathrm{PD}$, the cholesterol esterifying activity is reduced in fibroblasts and specific ChoE are reduced in the visual cortex ${ }^{42,43}$. Based on data obtained using several PD-related biological systems, it is not evident whether modulation of specific ChoE metabolic events may have a protective or pathological impact ${ }^{44,45}$. Phosphatidylcholine (PC), the most abundant glycerophospholipid in membranes is involved in the control of inflammation, neuronal differentiation and cholesterol homeostasis ${ }^{46-48}$. Our data pointed out an increment in almost the complete profile of PC at the level of CSF derived from PD subjects. However, decreased levels in multiple PC have been observed in plasma, frontal cortex and substantia nigra from PD patients 35,49,50. This tendency has been also observed in substantia nigra and brain tissue derived from a mouse model of PD and from MPTP-treated goldfish respectively 51,52. Morever, specific alpha-synuclein isoforms differentially interacts with PC membranes ${ }^{53-56}$. It has been proposed that different variation in ceramide (Cer) levels across brain areas may be linked to alpha-synuclein accumulation ${ }^{23}$. However, controversial data exist about the Cer plasma levels in PD patients 35,57 , 58. In general, an increment in Cer levels is commonly observed in different studies performed in PD animal and cellular models ${ }^{59-62}$. However, the consequences associated to Cer increment are not fully understood, being potentially detrimental or beneficial for different PD-related mechanisms. Sphingomyelin (SM), a major myelin component, is considered a source of bioactive lipidic molecules which play a role in inflammation, autophagy and cell death ${ }^{63-66}$. According to our data obtained in CSF, SM accumulation has been also observed in: i) LB aggregates ${ }^{67}$, ii) primary visual cortex from PD subjects as well as in substantia nigra from males with PD 42,50 and iii) PD patients with sphingomyelinase-1 mutations (risk factor) ${ }^{68,69}$. Although multiple factors suggest a potential 
role of SM accumulation in PD-associated neurodegeneration, more experimental evidences are needed to further elucidate the concise function of SM not only in alpha-synuclein aggregation, but also in inflammatory balance.

It is important to note that our data obtained at the level of CSF partially corroborate previous associations between PD and the levels of fatty acils, glycerolipids, glycerophospholipids, sphingolipids and sterols. Moreover, our pilot study establishes novel links between primary fatty amides (FAA) and N-acyl ethanolamines (NAE) with PD. However, although our untargeted lipidomic work has uncovered many intricacies in the CSF lipidomic homeostasis in the context of PD, there are potential limitations of our study that warrant discussion. First, due to the technological approach used, we failed to accurately monitor many lipid species present at low levels that might also participate in PD pathophysiology. Second, and based on the current knowledge, it is unclear whether the CSF lipid imbalance observed reflects pathological or compensatory mechanisms. Third, our study does not consider the effect of variables such as sex, age, PD etiology and/or mutational profiles. Finally, this study should be validated and complemented with additional patient cohorts with the aim to potentiate the deployment of biofluid lipid-based studies in paired CSF and plasma samples to identify potential biomarkers and/or druggable targets to be monitored across PD and other atypical parkinsonisms.

\title{
5. Conclusions
}

A CSF lipidomic approach performed in PD and control subjects $(n=30)$ has detected 257 metabolic features by ultra-high performance liquid chromatography - mass spectrometry (UHPLC-MS). A supervised OPLS model showed a clear separation between control and PD subjects indicating that the lipids responsible for this separation were mainly glycerolipids (MAG, DAG, TAG), fatty acids (SFA, MUFA), primary fatty amides, glycerophospholipids (PC, PE) and sphingolipids (Cer, SM), which were increased in PD group. Univariate data analysis also revealed a general increase in the CSF lipid metabolic profile in PD. Overall, these results suggest that multiple CSF lipid species tend to be increased in PD compared to control subjects. However, the predictive ability of the OPLS model was low, indicating that further follow-up studies are needed to validate this lipidomic signature in large CSF cohorts with the aim to potentially use CSF lipids as discriminatory biomarkers in terms of sensitivity and specificity.

\begin{abstract}
Abbreviations. AC: Acyl carnitines; BA: Bile acids; Cer: Ceramides; ChoE: Cholesteryl esters; DAG: Diacylglycerides; FAA: Fatty acid amides (Primary Fatty Amides); LPE: Lysophosphatidylethanolamines; LPI: Lysophosphatidylinositols; LPC: Lysophosphatidylcholines; MAG: Monoacylglycerides; MUFA: Monounsaturated fatty acids; NAE: N-acyl ethanolamines; NEFA: Non-esterified fatty acids; OPLS: Orthogonal partial least-squares to latent structures; PC: Phosphatidylcholines; PCA: Principal Component Analysis; PE: Phosphatidylethanolamines; PUFA: Polyunsaturated fatty acids; SFA: Saturated fatty acids; SM: Sphingomyelins; TAG: Triacylglycerides; UFA: Unsaturated fatty acids.
\end{abstract}

Supplementary Materials: The following are available online at www.mdpi.com/xxx/s1 Supplementary Table 1: "Raw data per metabolite_1" and "Raw data per chemical class_1" contain raw intensity data per metabolites and per metabolite class of the untransformed data, respectively. "Raw data per metabolite_2" and "Raw data per chemical class_2" contain raw intensity data per metabolites and per metabolite class of the data after square root transformation of the data. These sheets also include: i) "Individual notation" refers to the confirmed identification of the metabolites. Overlapping of two or more metabolites or non-confirmed identification is indicated in "Individual composition (or probable ID)", ii) Average group intensities and standard errors, iii) Shapiro test: used for testing the normality of data (Shapiro test (p) row is marked in red if sample comes from normally distributed population), iv) Fold-changes and unpaired Student's t-test p-values (or Welch's t test where unequal variances were found) for the comparison PD vs. control and v) "Heatmap" contains the metabolites' identification code, log2(fold-changes) and unpaired Student's t-test p-values illustrated in the heatmap. 
Author Contributions: Conceptualization, Enrique Santamaría.; software, Enrique Santamaría, Joaquín Fernández-Irigoyen.; formal analysis, Enrique Santamaría, Joaquín Fernández-Irigoyen, Paz Cartas-Cejudo; investigation, Enrique Santamaría, Joaquín Fernández-Irigoyen, Paz Cartas-Cejudo; resources, Enrique Santamaría, Joaquín Fernández-Irigoyen; funding acquisition, Enrique Santamaría, Joaquín Fernández-Irigoyen; writing-original draft preparation, Enrique Santamaría. All authors have read and agreed to the published version of the manuscript.

Funding: This work was funded by grants from the Spanish Ministry of Science Innovation and Universities (Ref. PID2019-110356RB-I00 to JF-I and ES) and the Department of Economic and Business Development from Government of Navarra (Ref. 0011-1411-2020-000028 to ES).

Institutional Review Board Statement: The study was conducted in accordance with the Declaration of Helsinki of 1975 (revised in 2013) and all assessments, post-mortem evaluations, and procedures were previously approved by the Local Clinical Ethics Committee (protocol code: 2016/36). Cerebrospinal fluid (CSF) samples and associated clinical and neuropathological data from patients with PD were supplied by the Parkinson's UK Brain Bank, funded by Parkinson's UK, a charity registered in England and Wales (258197) and in Scotland (SC037554).

Informed Consent Statement: According to the Spanish Law 14/2007 of Biomedical Research, inform written consent forms were obtained for research purposes from relatives of patients included in this study.

Data Availability Statement: Data available on request from the authors.

Acknowledgments: The authors are very grateful to the patients who generously donated the brain tissue and fluid samples for research purposes. The authors thank the collaboration of Parkinson's UK Brain Bank funded by Parkinson's UK, a charity registered in England and Wales (258197) and in Scotland (SC037554). The authors want to kindly thank Djordje Gveric (Centre for Brain Sciences, Imperial College London, London, UK) and technical personnel from OWL Metabolomics company (Derio, Spain) for their help in the management of associated clinical, neuropathological and molecular data. The Proteomics Unit of Navarrabiomed is a member of ProteoRed and PRB3-ISCIII and is supported by grant PT17/0019, of the PE I+D+I 2013-2016, funded by ISCIII and ERDF.

Conflicts of Interest: The authors declare no conflict of interest.

\section{Appendix A}

Table A1. Internal Standard Solutions Platform 1.

\begin{tabular}{|c|c|c|c|c|}
\hline IS & $\begin{array}{l}\text { IS Stock Solu- } \\
\text { tion }(\mu \mathrm{g} / \mathrm{mL})\end{array}$ & IS Stock Solution & $\begin{array}{l}\text { IS Intermedi- } \\
\text { ate Solution in } \\
\mathrm{CHCl}_{3}: \mathrm{MeOH} \\
(2: 1)(\mu \mathrm{g} / \mathrm{mL})\end{array}$ & $\begin{array}{c}\text { IS Working Solu- } \\
\text { tion in } \mathrm{MeOH} \\
(\mu \mathrm{g} / \mathrm{mL})\end{array}$ \\
\hline 13:0 Lyso PC & 10000 & $\mathrm{CHCl}_{3}$ & 10 & 0.1 \\
\hline $\begin{array}{l}\text { Dehydrocholic } \\
\text { acid }\end{array}$ & 5000 & $\mathrm{CHCl}_{3}: \mathrm{MeOH}(1: 1)$ & 30 & 0.3 \\
\hline $\begin{array}{c}\text { Nonadecanoic } \\
\text { acid }\end{array}$ & 10000 & $\mathrm{CHCl}_{3}$ & 500 & 5.0 \\
\hline $\begin{array}{c}\text { Trypto- } \\
\text { phan-(indole-d } \\
5 \text { ) }\end{array}$ & 5000 & $\begin{array}{c}0.05 \% \text { Formic acid } \\
\text { in water }\end{array}$ & 200 & 2.0 \\
\hline
\end{tabular}


Table A2. Internal Standard Solutions Platform 2.

\begin{tabular}{cccc}
\hline IS & $\begin{array}{c}\text { IS Stock } \\
\text { Solution } \\
(\boldsymbol{\mu g} / \mathbf{m L})\end{array}$ & IS Stock Solution & $\begin{array}{c}\text { Working IS Solution } \\
\mathrm{CHCl}_{3}: \mathbf{M e O H}(\mathbf{2 : 1 )}(\boldsymbol{\mu g} / \mathbf{m L})\end{array}$ \\
\hline SM (d18:1/6:0) & 5000 & $\mathrm{CHCl}_{3}$ & 5 \\
\hline PE (17:0/17:0) & 10000 & $\mathrm{CHCl}_{3}:{\mathrm{MeOH}: \mathrm{H}_{2} \mathrm{O}}$ & 50 \\
\hline PC (19:0/19:0) & 10000 & $\mathrm{CHCl}_{3}$ & 10 \\
\hline TG (13:0/13:0/13:0) & 10000 & $\mathrm{CHCl}_{3}$ & 5 \\
\hline TG (17:0/17:0/17:0) & 10000 & $\mathrm{CHCl}_{3}$ & 10 \\
\hline Cer(d18:1/17:0) & 10000 & $\mathrm{CHCl}_{3}$ & 250 \\
\hline ChoE(12:0) & 10000 & $\mathrm{CHCl}_{3}$ & 5 \\
\hline
\end{tabular}

Multivariate data analysis of all CSF samples, pool samples and quality control (QC) samples was initially performed. Score scatter plot corresponding to PCA analysis of these samples is shown in Figure 2. Proximity and overlap of the Pool and QC injections provides a good indication of the reproducibility and quality of the measurements.

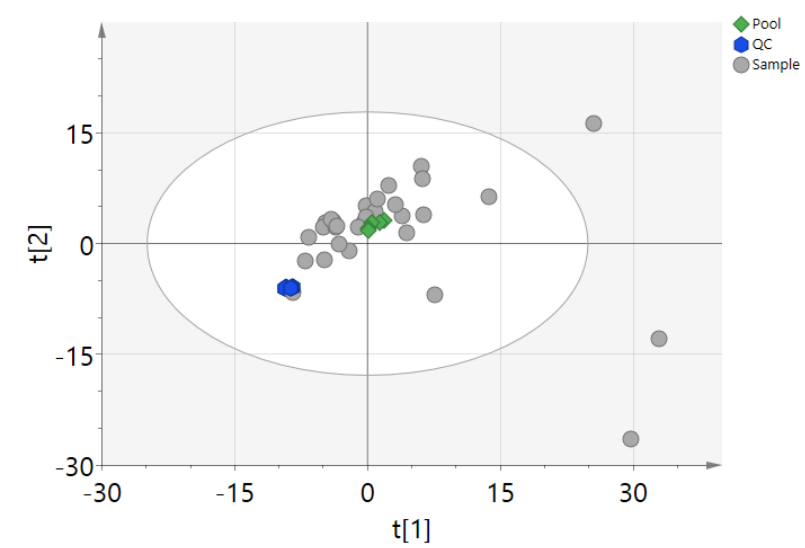

Figure A1. Score scatter plot of the PCA model of CSF, Pool and QC samples. Pool: $15 \mu$ of each CSF sample were collected and pooled together. Model diagnostics ( $A=6, R 2 X=0.829, Q 2 X=0.496)$.

After validating the quality of the experiment, the Pool and QC injections were removed from the analysis and a score scatter plot of the PCA model of all cerebrospinal fluid samples was generated.

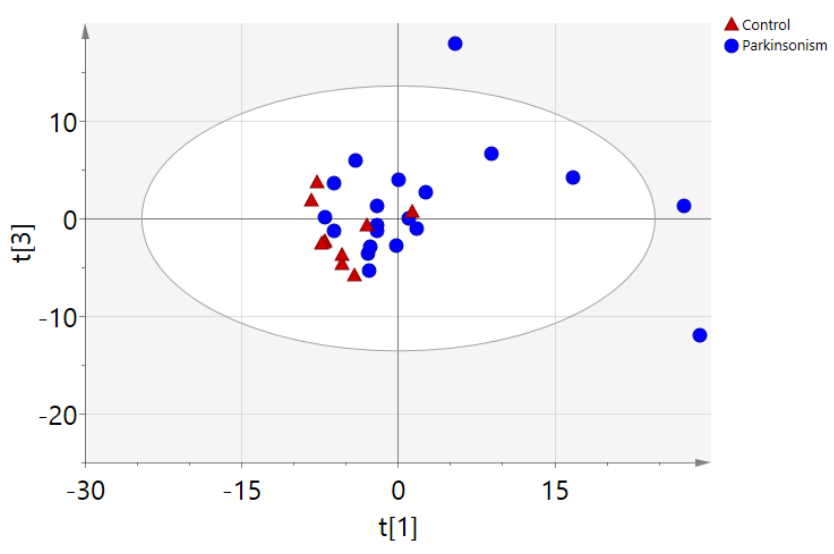

Figure A2. Score scatter plot of the PCA model of cerebrospinal fluid samples. Model diagnostics $(\mathrm{A}=6, \mathrm{R} 2 \mathrm{X}=0.807, \mathrm{Q} 2 \mathrm{X}=0.401)$.

Shapiro-Wilk test was used for testing the normality of data (results included in Supplementary Table 1), revealing that the majority of the metabolites measured in the 
CSF samples from PD patients were not following a normal distribution. Then, Box-Cox method for correcting non-normally distributed data by variable transformations was applied, identifying the square root transformation as the optimal for most of the metabolites. This kind of transformations are a common pre-treatment method in metabolomics for the conversion of the data, which correct aspects that hinder the biological interpretation of data sets by emphasizing the biological information and thus, improving their physiological interpretability. Score scatter plot corresponding to PCA analysis of CSF samples after square root transformation of the data is shown in Figure A3.

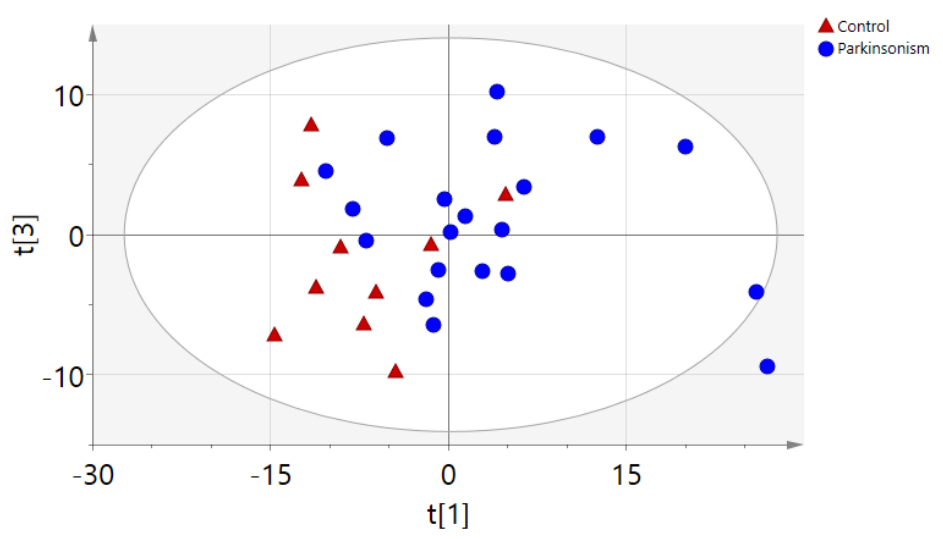

Figure A3. Score scatter plot of the PCA model of cerebrospinal fluid samples after square root transformation of the data. Model diagnostics $(A=4, R 2 X=0.752, Q 2 X=0.505)$.

This Score scatter plot showed certain clustering of samples according to the presence or absence of the disease and identified sample PD353 as a potential outlier since it appeared outside the Hotelling's T2 ellipse. Following Chauvenet's criterion, further inspection of the data relating to this sample revealed that it presented elevated levels of sphingolipids compared to the rest of the samples from PD group. However, the levels of the majority of the metabolites in this sample were similar to those of the samples from the same group and thus, it was not excluded from the multivariate and univariate analyses. Metabolites responsible for this certain separation observed between CSF samples of PD and control subjects can be observed in the loadings scatter plot (Figure A4), which is a graph related to the score scatter plot shown in Figure A3.

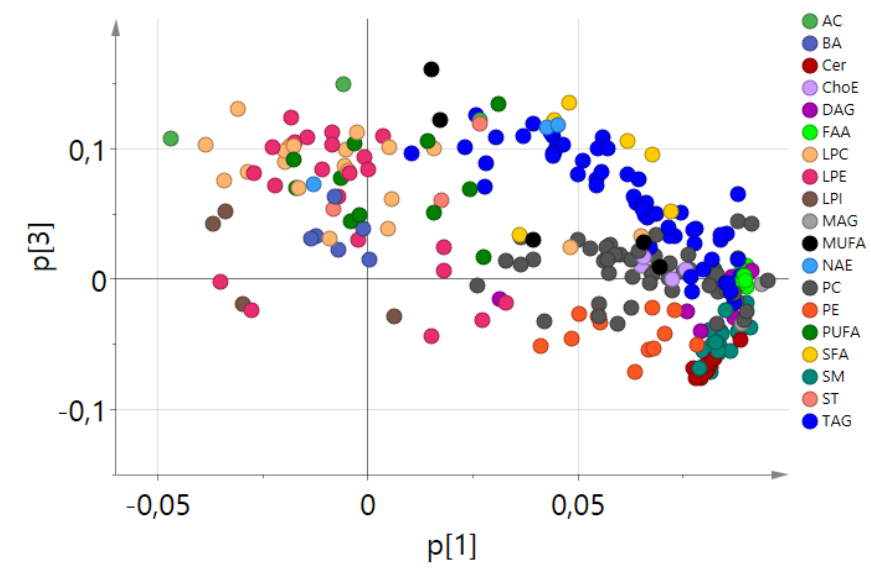

Figure A4. Loadings scatter plot of the PCA model of cerebrospinal fluid samples after square root transformation of the data. Model diagnostics ( $A=4, R 2 X=0.752, Q 2 X=0.505)$.

Lipids lying away from the plot origin have stronger impact on the model; besides, variables positively correlated are grouped together, while variables negatively correlated are positioned in the opposite sides of the plot origin. In this case, the metabolites 
responsible for the differences observed were mainly glycerolipids (monoacylglycerols -MAG-, diacylglycerols -DAG-, triacylglycerols -TAG-), fatty acids (saturated fatty acids -SFA-, monounsaturated fatty acids -MUFA-), primary fatty amides (FAA), glycerophospholipids (phosphatidylcholines -PC-, phosphatidylethanolamines -PE-) and sphingolipids (ceramides -Cer-, sphingomyelins -SM-), which were increased in PD compared to control group; and lysoglycerophospholipids (lysophosphatidylcholines -LPC-, lysophosphatidylethanolamines -LPE-, lyshophosphatidylinositols -LPI-) and bile acids (BA), which seemed to be increased in controls compared to PD group.

\section{References}

1. Shevchenko, A.; Simons, K., Lipidomics: Coming to grips with lipid diversity. Nat Rev Mol Cell Biol 2010, 11, (8), 593-598.

2. Brugger, B., Lipidomics: Analysis of the lipid composition of cells and subcellular organelles by electrospray ionization mass spectrometry. Annu Rev Biochem 2014, 83, 79-98.

3. Piomelli, D.; Astarita, G.; Rapaka, R., A neuroscientist's guide to lipidomics. Nat Rev Neurosci 2007, 8, (10), 743-754.

4. Gross, R.W.; Han, X., Lipidomics at the interface of structure and function in systems biology. Chem Biol 2011, 18, (3), $284-291$.

5. Lauwers, E.; Goodchild, R.; Verstreken, P., Membrane Lipids in Presynaptic Function and Disease. Neuron 2016, 90, (1), 11-25.

6. Klemann, C.; Martens, G.J.M.; Sharma, M.; Martens, M.B.; Isacson, O.; Gasser, T.; Visser, J.E.; Poelmans, G., Integrated molecular landscape of Parkinson's disease. NPJ Parkinsons Dis 2017, 3, 14.

7. Sidransky, E.; Lopez, G., The link between the GBA gene and parkinsonism. Lancet Neurol 2012, 11, (11), $986-998$.

8. Do, C.B.; Tung, J.Y.; Dorfman, E.; Kiefer, A.K.; Drabant, E.M.; Francke, U.; Mountain, J.L.; Goldman, S.M.; Tanner, C.M.; Langston, J.W.; Wojcicki, A.; Eriksson, N., Web-based genome-wide association study identifies two novel loci and a substantial genetic component for Parkinson's disease. PLoS Genet 2011, 7, (6), e1002141.

9. Pankratz, N.; Wilk, J.B.; Latourelle, J.C.; DeStefano, A.L.; Halter, C.; Pugh, E.W.; Doheny, K.F.; Gusella, J.F.; Nichols, W.C.; Foroud, T.; Myers, R.H., Genomewide association study for susceptibility genes contributing to familial Parkinson disease. Hum Genet 2009, 124, (6), 593-605.

10. Robak, L.A.; Jansen, I.E.; van Rooij, J.; Uitterlinden, A.G.; Kraaij, R.; Jankovic, J.; Heutink, P.; Shulman, J.M., Excessive burden of lysosomal storage disorder gene variants in Parkinson's disease. Brain 2017, 140, (12), 3191-3203.

11. Galvagnion, C., The Role of Lipids Interacting with alpha-Synuclein in the Pathogenesis of Parkinson's Disease. J Parkinsons Dis 2017, 7, (3), 433-450.

12. Shahmoradian, S.H.; Lewis, A.J.; Genoud, C.; Hench, J.; Moors, T.E.; Navarro, P.P.; Castano-Diez, D.; Schweighauser, G.; Graff-Meyer, A.; Goldie, K.N.; Sutterlin, R.; Huisman, E.; Ingrassia, A.; Gier, Y.; Rozemuller, A.J.M.; Wang, J.; Paepe, A.; Erny, J.; Staempfli, A.; Hoernschemeyer, J.; Grosseruschkamp, F.; Niedieker, D.; El-Mashtoly, S.F.; Quadri, M.; Van, I.W.F.J.; Bonifati, V.; Gerwert, K.; Bohrmann, B.; Frank, S.; Britschgi, M.; Stahlberg, H.; Van de Berg, W.D.J.; Lauer, M.E., Lewy pathology in Parkinson's disease consists of crowded organelles and lipid membranes. Nat Neurosci 2019, 22, (7), 1099-1109.

13. Seyer, A.; Boudah, S.; Broudin, S.; Junot, C.; Colsch, B., Annotation of the human cerebrospinal fluid lipidome using high resolution mass spectrometry and a dedicated data processing workflow. Metabolomics 2016, 12, 91.

14. Naudi, A.; Cabre, R.; Jove, M.; Ayala, V.; Gonzalo, H.; Portero-Otin, M.; Ferrer, I.; Pamplona, R., Lipidomics of human brain aging and Alzheimer's disease pathology. Int Rev Neurobiol 2015, 122, 133-189.

15. Consortium, L.S.I., Lipidomics needs more standardization. Nat Metab 2019, 1, (8), 745-747.

16. Proitsi, P.; Kim, M.; Whiley, L.; Simmons, A.; Sattlecker, M.; Velayudhan, L.; Lupton, M.K.; Soininen, H.; Kloszewska, I.; Mecocci, P.; Tsolaki, M.; Vellas, B.; Lovestone, S.; Powell, J.F.; Dobson, R.J.; Legido-Quigley, C., Association of blood lipids with Alzheimer's disease: A comprehensive lipidomics analysis. Alzheimers Dement 2017, 13, (2), 140-151.

17. Wong, M.W.; Braidy, N.; Poljak, A.; Pickford, R.; Thambisetty, M.; Sachdev, P.S., Dysregulation of lipids in Alzheimer's disease and their role as potential biomarkers. Alzheimers Dement 2017, 13, (7), 810-827.

18. Gonzalo, H.; Brieva, L.; Tatzber, F.; Jove, M.; Cacabelos, D.; Cassanye, A.; Lanau-Angulo, L.; Boada, J.; Serrano, J.C.; Gonzalez, C.; Hernandez, L.; Peralta, S.; Pamplona, R.; Portero-Otin, M., Lipidome analysis in multiple sclerosis reveals protein lipoxidative damage as a potential pathogenic mechanism. J Neurochem 2012, 123, (4), 622-634.

19. Pieragostino, D.; Cicalini, I.; Lanuti, P.; Ercolino, E.; di Ioia, M.; Zucchelli, M.; Zappacosta, R.; Miscia, S.; Marchisio, M.; Sacchetta, P.; Onofrj, M.; Del Boccio, P., Enhanced release of acid sphingomyelinase-enriched exosomes generates a lipidomics signature in CSF of Multiple Sclerosis patients. Sci Rep 2018, 8, (1), 3071.

20. Blasco, H.; Veyrat-Durebex, C.; Bocca, C.; Patin, F.; Vourc'h, P.; Kouassi Nzoughet, J.; Lenaers, G.; Andres, C.R.; Simard, G.; Corcia, P.; Reynier, P., Lipidomics Reveals Cerebrospinal-Fluid Signatures of ALS. Sci Rep 2017, 7, (1), 17652.

21. Barr, J.; Vazquez-Chantada, M.; Alonso, C.; Perez-Cormenzana, M.; Mayo, R.; Galan, A.; Caballeria, J.; Martin-Duce, A.; Tran, A.; Wagner, C.; Luka, Z.; Lu, S.C.; Castro, A.; Le Marchand-Brustel, Y.; Martinez-Chantar, M.L.; Veyrie, N.; Clement, K.; Tordjman, J.; Gual, P.; Mato, J.M., Liquid chromatography-mass spectrometry-based parallel metabolic profiling of human and mouse model serum reveals putative biomarkers associated with the progression of nonalcoholic fatty liver disease. J Proteome Res 2010, 9, (9), 4501-4512.

22. Fonteh, A.N.; Harrington, R.J.; Huhmer, A.F.; Biringer, R.G.; Riggins, J.N.; Harrington, M.G., Identification of disease markers in human cerebrospinal fluid using lipidomic and proteomic methods. Dis Markers 2006, 22, (1-2), 39-64. 
23. Xicoy, H.; Wieringa, B.; Martens, G.J.M., The Role of Lipids in Parkinson's Disease. Cells 2019, 8, (1).

24. Kamel, F.; Goldman, S.M.; Umbach, D.M.; Chen, H.; Richardson, G.; Barber, M.R.; Meng, C.; Marras, C.; Korell, M.; Kasten, M.; Hoppin, J.A.; Comyns, K.; Chade, A.; Blair, A.; Bhudhikanok, G.S.; Webster Ross, G.; William Langston, J.; Sandler, D.P.; Tanner, C.M., Dietary fat intake, pesticide use, and Parkinson's disease. Parkinsonism Relat Disord 2014, $20,(1), 82-87$.

25. Fabelo, N.; Martin, V.; Santpere, G.; Marin, R.; Torrent, L.; Ferrer, I.; Diaz, M., Severe alterations in lipid composition of frontal cortex lipid rafts from Parkinson's disease and incidental Parkinson's disease. Mol Med 2011, 17, (9-10), 1107-1118.

26. Julien, C.; Berthiaume, L.; Hadj-Tahar, A.; Rajput, A.H.; Bedard, P.J.; Di Paolo, T.; Julien, P.; Calon, F., Postmortem brain fatty acid profile of levodopa-treated Parkinson disease patients and parkinsonian monkeys. Neurochem Int 2006, 48, (5), $404-414$.

27. Schmid, S.P.; Schleicher, E.D.; Cegan, A.; Deuschle, C.; Baur, S.; Hauser, A.K.; Synofzik, M.; Srulijes, K.; Brockmann, K.; Berg, D.; Maetzler, W., Cerebrospinal fluid fatty acids in glucocerebrosidase-associated Parkinson's disease. Mov Disord 2012, 27, (2), 288-292.

28. Abbott, S.K.; Jenner, A.M.; Spiro, A.S.; Batterham, M.; Halliday, G.M.; Garner, B., Fatty acid composition of the anterior cingulate cortex indicates a high susceptibility to lipid peroxidation in Parkinson's disease. J Parkinsons Dis 2015, 5, (1), 175-185.

29. Ben Gedalya, T.; Loeb, V.; Israeli, E.; Altschuler, Y.; Selkoe, D.J.; Sharon, R., Alpha-synuclein and polyunsaturated fatty acids promote clathrin-mediated endocytosis and synaptic vesicle recycling. Traffic 2009, 10, (2), 218-234.

30. Sharon, R.; Bar-Joseph, I.; Frosch, M.P.; Walsh, D.M.; Hamilton, J.A.; Selkoe, D.J., The formation of highly soluble oligomers of alpha-synuclein is regulated by fatty acids and enhanced in Parkinson's disease. Neuron 2003, 37, (4), 583-595.

31. Perrin, R.J.; Woods, W.S.; Clayton, D.F.; George, J.M., Exposure to long chain polyunsaturated fatty acids triggers rapid multimerization of synucleins. J Biol Chem 2001, 276, (45), 41958-41962.

32. Tu-Sekine, B.; Goldschmidt, H.; Raben, D.M., Diacylglycerol, phosphatidic acid, and their metabolic enzymes in synaptic vesicle recycling. Adv Biol Regul 2015, 57, 147-152.

33. Almena, M.; Merida, I., Shaping up the membrane: Diacylglycerol coordinates spatial orientation of signaling. Trends Biochem Sci 2011, 36, (11), 593-603.

34. Ahmadian, M.; Duncan, R.E.; Jaworski, K.; Sarkadi-Nagy, E.; Sul, H.S., Triacylglycerol metabolism in adipose tissue. Future Lipidol 2007, 2, (2), 229-237.

35. Zhang, J.; Zhang, X.; Wang, L.; Yang, C., High Performance Liquid Chromatography-Mass Spectrometry (LC-MS) Based Quantitative Lipidomics Study of Ganglioside-NANA-3 Plasma to Establish Its Association with Parkinson's Disease Patients. Med Sci Monit 2017, 23, 5345-5353.

36. Chan, R.B.; Perotte, A.J.; Zhou, B.; Liong, C.; Shorr, E.J.; Marder, K.S.; Kang, U.J.; Waters, C.H.; Levy, O.A.; Xu, Y.; Shim, H.B.; Pe'er, I.; Di Paolo, G.; Alcalay, R.N., Elevated GM3 plasma concentration in idiopathic Parkinson's disease: A lipidomic analysis. PLoS ONE 2017, 12, (2), e0172348.

37. Vikdahl, M.; Backman, L.; Johansson, I.; Forsgren, L.; Haglin, L., Cardiovascular risk factors and the risk of Parkinson's disease. Eur J Clin Nutr 2015, 69, (6), 729-733.

38. He, Q.; Wang, M.; Petucci, C.; Gardell, S.J.; Han, X., Rotenone induces reductive stress and triacylglycerol deposition in C2C12 cells. Int J Biochem Cell Biol 2013, 45, (12), 2749-2755.

39. Sere, Y.Y.; Regnacq, M.; Colas, J.; Berges, T., A Saccharomyces cerevisiae strain unable to store neutral lipids is tolerant to oxidative stress induced by alpha-synuclein. Free Radic Biol Med 2010, 49, (11), 1755-1764.

40. Spann, N.J.; Glass, C.K., Sterols and oxysterols in immune cell function. Nat Immunol 2013, 14, (9), 893-900.

41. Hannich, J.T.; Umebayashi, K.; Riezman, H., Distribution and functions of sterols and sphingolipids. Cold Spring Harb Perspect Biol 2011, 3, (5).

42. Cheng, D.; Jenner, A.M.; Shui, G.; Cheong, W.F.; Mitchell, T.W.; Nealon, J.R.; Kim, W.S.; McCann, H.; Wenk, M.R.; Halliday, G.M.; Garner, B., Lipid pathway alterations in Parkinson's disease primary visual cortex. PLoS ONE 2011, 6, (2), e17299.

43. Musanti, R.; Parati, E.; Lamperti, E.; Ghiselli, G., Decreased cholesterol biosynthesis in fibroblasts from patients with Parkinson disease. Biochem Med Metab Biol 1993, 49, (2), 133-142.

44. Magalhaes, J.; Gegg, M.E.; Migdalska-Richards, A.; Doherty, M.K.; Whitfield, P.D.; Schapira, A.H., Autophagic lysosome reformation dysfunction in glucocerebrosidase deficient cells: Relevance to Parkinson disease. Hum Mol Genet 2016, 25, (16), 3432-3445.

45. Zhang, S.; Glukhova, S.A.; Caldwell, K.A.; Caldwell, G.A., NCEH-1 modulates cholesterol metabolism and protects against alpha-synuclein toxicity in a C. elegans model of Parkinson's disease. Hum Mol Genet 2017, 26, (19), 3823-3836.

46. Treede, I.; Braun, A.; Sparla, R.; Kuhnel, M.; Giese, T.; Turner, J.R.; Anes, E.; Kulaksiz, H.; Fullekrug, J.; Stremmel, W.; Griffiths, G.; Ehehalt, R., Anti-inflammatory effects of phosphatidylcholine. J Biol Chem 2007, 282, (37), 27155-27164.

47. Lagace, T.A., Phosphatidylcholine: Greasing the Cholesterol Transport Machinery. Lipid Insights 2015, 8, (Suppl 1), 65-73.

48. Marcucci, H.; Paoletti, L.; Jackowski, S.; Banchio, C., Phosphatidylcholine biosynthesis during neuronal differentiation and its role in cell fate determination. J Biol Chem 2010, 285, (33), 25382-25393.

49. Wood, P.L.; Tippireddy, S.; Feriante, J.; Woltjer, R.L., Augmented frontal cortex diacylglycerol levels in Parkinson's disease and Lewy Body Disease. PLoS ONE 2018, 13, (3), e0191815.

50. Seyfried, T.N.; Choi, H.; Chevalier, A.; Hogan, D.; Akgoc, Z.; Schneider, J.S., Sex-Related Abnormalities in Substantia Nigra Lipids in Parkinson's Disease. ASN Neuro 2018, 10, 1759091418781889.

51. Farmer, K.; Smith, C.A.; Hayley, S.; Smith, J., Major Alterations of Phosphatidylcholine and Lysophosphotidylcholine Lipids in the Substantia Nigra Using an Early Stage Model of Parkinson's Disease. Int J Mol Sci 2015, 16, (8), $18865-18877$. 
52. Lu, Z.; Wang, J.; Li, M.; Liu, Q.; Wei, D.; Yang, M.; Kong, L., (1)H NMR-based metabolomics study on a goldfish model of Parkinson's disease induced by 1-methyl-4-phenyl-1,2,3,6-tetrahydropyridine (MPTP). Chem Biol Interact 2014, $223,18-26$.

53. Stockl, M.; Fischer, P.; Wanker, E.; Herrmann, A., Alpha-synuclein selectively binds to anionic phospholipids embedded in liquid-disordered domains. J Mol Biol 2008, 375, (5), 1394-1404.

54. Jiang, Z.; de Messieres, M.; Lee, J.C., Membrane remodeling by alpha-synuclein and effects on amyloid formation. J Am Chem Soc 2013, 135, (43), 15970-15973.

55. Di Pasquale, E.; Fantini, J.; Chahinian, H.; Maresca, M.; Taieb, N.; Yahi, N., Altered ion channel formation by the Parkinson's-disease-linked E46K mutant of alpha-synuclein is corrected by GM3 but not by GM1 gangliosides. J Mol Biol 2010, 397, (1), 202-218.

56. O'Leary, E.I.; Jiang, Z.; Strub, M.P.; Lee, J.C., Effects of phosphatidylcholine membrane fluidity on the conformation and aggregation of N-terminally acetylated alpha-synuclein. J Biol Chem 2018, 293, (28), 11195-11205.

57. Mielke, M.M.; Maetzler, W.; Haughey, N.J.; Bandaru, V.V.; Savica, R.; Deuschle, C.; Gasser, T.; Hauser, A.K.; Graber-Sultan, S.; Schleicher, E.; Berg, D.; Liepelt-Scarfone, I., Plasma ceramide and glucosylceramide metabolism is altered in sporadic Parkinson's disease and associated with cognitive impairment: A pilot study. PLoS ONE 2013, 8, (9), e73094.

58. Atashrazm, F.; Hammond, D.; Perera, G.; Dobson-Stone, C.; Mueller, N.; Pickford, R.; Kim, W.S.; Kwok, J.B.; Lewis, S.J.G.; Halliday, G.M.; Dzamko, N., Reduced glucocerebrosidase activity in monocytes from patients with Parkinson's disease. Sci Rep 2018, 8, (1), 15446.

59. Lupescu, A.; Jilani, K.; Zbidah, M.; Lang, F., Induction of apoptotic erythrocyte death by rotenone. Toxicology 2012, 300, (3), 132-137.

60. Lin, G.; Lee, P.T.; Chen, K.; Mao, D.; Tan, K.L.; Zuo, Z.; Lin, W.W.; Wang, L.; Bellen, H.J., Phospholipase PLA2G6, a Parkinsonism-Associated Gene, Affects Vps26 and Vps35, Retromer Function, and Ceramide Levels, Similar to alpha-Synuclein Gain. Cell Metab 2018, 28, (4), 605-618 e6.

61. Ferrazza, R.; Cogo, S.; Melrose, H.; Bubacco, L.; Greggio, E.; Guella, G.; Civiero, L.; Plotegher, N., LRRK2 deficiency impacts ceramide metabolism in brain. Biochem Biophys Res Commun 2016, 478, (3), 1141-1146.

62. Torres-Odio, S.; Key, J.; Hoepken, H.H.; Canet-Pons, J.; Valek, L.; Roller, B.; Walter, M.; Morales-Gordo, B.; Meierhofer, D.; Harter, P.N.; Mittelbronn, M.; Tegeder, I.; Gispert, S.; Auburger, G., Progression of pathology in PINK1-deficient mouse brain from splicing via ubiquitination, ER stress, and mitophagy changes to neuroinflammation. J Neuroinflammation 2017, 14, (1), 154.

63. Nixon, G.F., Sphingolipids in inflammation: Pathological implications and potential therapeutic targets. Br J Pharmacol 2009, 158, (4), 982-993.

64. Norris, G.H.; Blesso, C.N., Dietary and Endogenous Sphingolipid Metabolism in Chronic Inflammation. Nutrients 2017, 9, (11).

65. Kiraz, Y.; Adan, A.; Kartal Yandim, M.; Baran, Y., Major apoptotic mechanisms and genes involved in apoptosis. Tumour Biol 2016, 37, (7), 8471-8486.

66. Tommasino, C.; Marconi, M.; Ciarlo, L.; Matarrese, P.; Malorni, W., Autophagic flux and autophagosome morphogenesis require the participation of sphingolipids. Apoptosis 2015, 20, (5), 645-657.

67. den Jager, W.A., Sphingomyelin in Lewy inclusion bodies in Parkinson's disease. Arch Neurol 1969, $21,(6), 615-619$.

68. Foo, J.N.; Liany, H.; Bei, J.X.; Yu, X.Q.; Liu, J.; Au, W.L.; Prakash, K.M.; Tan, L.C.; Tan, E.K., Rare lysosomal enzyme gene SMPD1 variant (p.R591C) associates with Parkinson's disease. Neurobiol Aging 2013, 34, (12), 2890 e13-5.

69. Mao, C.Y.; Yang, J.; Wang, H.; Zhang, S.Y.; Yang, Z.H.; Luo, H.Y.; Li, F.; Shi, M.; Liu, Y.T.; Zhuang, Z.P.; Du, P.; Wang, Y.H.; Shi, C.H.; Xu, Y.M., SMPD1 variants in Chinese Han patients with sporadic Parkinson's disease. Parkinsonism Relat Disord 2017, $34,59-61$. 